\title{
DOSAGE DU CALCIUM DANS LE LAIT
}

\author{
par \\ Jean PIEN \\ Président de la Commission Internationale d'Analyse du Lait
}

La Fédération Internationale de Laiterie se préoccupe, entre autres choses, de la normalisation sur le plan international des méthodes d'analyse chimique et bactériologique du lait et des produits laitiers.

Plus de 40 normes ont déjà été ainsi mises au point et publiées par les commissions spécialisées de la F.I.L.

Lorsque le besoin se fait sentir de mettre à l'étude telle ou telle méthode analytique, sur la demande par exemple de la F.A.O. ou des Comités nationaux de la F.I.L. ou de la F.I.L. elle-même, un Groupe de travail de quelques spécialistes de la question est créé et un rapporteur est désigné.

Le rapport est soumis à la discussion de la commission compé. tente puis, après approbation éventuelle, ce rapport est diffusé auprès des 29 pays affiliés à la F.I.L. qui présentent des observations de fond et de forme grâce auxquelles un nouveau rapport est rédigé et soumis à la Commission. Ce n'est qu'après l'approbation définitive par cette commission que le projet élaboré devient "Norme officielle internationale de la F.I.L.".

En ce qui concerne le dosage du calcium dans le lait nous avons eu l'honneur d'être désigné comme rapporteur et responsable d'un Groupe de travail comprenant notamment les professeurs H.W. Kay (D), E. Pijanowski (P), G. Schwarz (D), J.A.B. Smith (G B), P. Swartling (S).

Ce Groupe de travail, après une série de travaux préliminaires, a sélectionné un certain nombre de méthodes, puis nous a chargé de les expérimenter et d'en extraire celle qui nous paraîtrait la plus susceptible d'être normalisée.

Le présent rapport rend compte de nos études dans ce domaine.

$$
\text { ** } *
$$

Les méthodes proposées par le Groupe de travail étaient basées sur les principes suivants : 
1) Détermination directe dans le lait (c'est-à-dire par défécation à l'acide trichloracétique puis précipitation du calcium à l'état d'oxalate et dosage de l'oxalate par manganimétrie et par complexométrie).

2) Minéralisation par voie sèche aviec adjonction d'acétate de magnésium, dissolution des cendres, précipitation du calcium par l'oxalate et dosage de l'oxalate par manganimétrie et par complexométrie.

3) A ces deux groupes de déterminations, nous avons ajouté la minéralisation nitroperchlorique suivie de la précipitation du calcium à l'état d'oxalate et dosage de l'oxalate par manganimétrie et par complexométrie.

\section{$*^{*} *$ \\ PREMIÉRE PARTIE}

\section{Description sommaire des méthodes employées}

Les méthodes que nous avons utilisées et qui sont décrites ci-après résultent d'une sélection, après essais préliminaires, et d'une synthèse des éléments de plusieurs méthodes dont les principales sont les suivantes :

Méthode proposée par le professeur Schwarz (Doc. F.I.L. 1, 1965). Méthode proposée par le professeur Swartling (Doc. F.I.L. 2, 1965). Méthode Davies et White (J. Dairy Res., 1962, 29, 285).

I) Préparation de l'analyse (défécation ou minéralisation).

Les trois méthodes suivantes ont été utilisées :

1) Défécation trichloracétique.

Dans une fiole jaugée de $50 \mathrm{ml}$, peser avec précision environ $20 \mathrm{~g}$ de lait ou mesurer $20 \mathrm{ml}$ de lait (entier ou écrémé).

Ajouter en agitant une solution aqueuse d'acide trichloracétique à 20 p. 100 (poids/volume) et compléter à $50 \mathrm{ml}$ avec ce réactif. Agiter vigoureusement pendant quelques secondes. Laisser reposer $30 \mathrm{mn}$. Filtrer sur papier Whatman no 40 ou d'une porosité équivalente (par exemple: Durieux no 111).

Recueillir le filtrat qui doit être limpide.

2) Minéralisation par voie sèche (avec ou sans acétate de magnésium).

Dans une capsule de platine ou de quartz peser exactement environ $10 \mathrm{~g}$ de lait ou mesurer $10 \mathrm{ml}$ de lait (entier ou écrémé). 
Dans les cas où l'on aura à juger l'influence de l'acétate de magnésium, ajouter au contenu de la capsule $2 \mathrm{ml}$ d'une solution aqueuse d'acétate de magnésium à 10 p. 100 .

Evaporer à sec au bain-marie bouillant. Après dessiccation complète, calciner au four à moufle à une température comprise entre $500^{\circ} \mathrm{C}$ et $550^{\circ} \mathrm{C}$ jusqu'à obtention de cendres blanches.

Après refroidissement de la capsule, la couvrir avec un verre de montre, dissoudre les cendres dans quelques ml d'acide chlorhydrique (en principe $3 \mathrm{ml}$ de $\mathrm{HCl} \mathrm{N}$ ) diluer avec de l'eau, transvaser dans une fiole jaugée de $50 \mathrm{ml}$, rincer le verre de montre et la capsule, recueillir les eaux de lavage dans la fiole. Refroidir s'il y a lieu. Compléter à $50 \mathrm{ml}$ avee de l'eau. Mélanger. Filtrer. Recueillir le filtrat.

3) Minéralisation par voie humide.

Dans une fiole de microkjeldahl, peser exactement environ $2 \mathrm{~g}$ de lait ou mesurer $2 \mathrm{ml}$ de lait (entier ou écrémé).

Ajouter $2,5 \mathrm{ml}$ d'acide perchlorique à $72 \mathrm{p}$. $100(\mathrm{~d}=1,67)$ et $2,5 \mathrm{ml}$ d'acide nitrique concentré $(\mathrm{d}=1,40)$ et quelques perles de verre.

Faire bouillir doucement jusqu'à décoloration complète, puis pendant encore $20 \mathrm{mn}$ après la décoloration. Laisser refroidir.

\section{II) Séparation du calcium à l'état d'oxalate.}

Dans un tube de centrifugation cylindrique de $30 \mathrm{ml}$ de volume total, gradué approximativement à $20 \mathrm{ml}$, introduire :

- Soit $5 \mathrm{ml} \mathrm{du}$ filtrat de la défécation trichloracétique (= $2 \mathrm{~g}$ ou $2 \mathrm{ml}$ de lait) plus $5 \mathrm{ml}$ d'acide trichloracétique à $12 \mathrm{p} .100$.

- Soit $10 \mathrm{ml} \mathrm{du}$ filtrat de la solution acide des cendres $(=2 \mathrm{~g}$ ou $2 \mathrm{ml}$ de lait).

- Soit la totalité du liquide de minéralisation nitroperchlorique en ayant soin de rincer le microkjeldahl avec le minimum d'eau ( 3 fois 1 à $2 \mathrm{ml}$ d'eau) (= $2 \mathrm{~g}$ ou $2 \mathrm{ml}$ de lait).

Dans les trois cas, ajouter $2 \mathrm{ml}$ d'une solution aqueuse d'oxalate d'ammonium saturée à froid, 2 gouttes de rouge de méthyle à 0,05 p. 100 dans l'alcool à 95 p. 100 et $2 \mathrm{ml}$ d'acide acétique à 20 p. 100. Mélanger par agitation circulaire et ajouter peu à peu de l'ammoniaque au demi jusqu'à coloration jaune pâle puis quelques gouttes d'acide acétique à 20 p. 100 jusqu'à coloration rose.

Laisser reposer $4 \mathrm{~h}$ à la température ordinaire. Diluer à $20 \mathrm{ml}$ avec de l'eau.

Centrifuger $10 \mathrm{mn}$ à $1400 \mathrm{G}$.

Décanter le liquide surnageant avec un dispositif à succion muni d'un tube capillaire.

Introduire dans le tube de centrifugation, pour rincer les parois, 
(mais sans remettre en suspension le culot d'oxalate de calcium) $5 \mathrm{ml}$ de solution d'ammoniaque à $2 \mathrm{p}$. 100 . Centrifuger $5 \mathrm{mn}$ à $1400 \mathrm{~g}$. Enlever le liquide surnageant. Faire ainsi trois lavages.

(Ces lavages ne sont nécessaires que lorsque le dosage se fera par manganimètrie. Ils sont inutiles dans le cas du dosage par complexométrie.)

\section{III) Dosage du calcium.}

Les deux méthodes suivantes ont été utilisées:

\section{1) Manganimétrie.}

Sur le culot d'oxalate de calcium, ajouter $2 \mathrm{ml}$ d'une solution d'acide sulfurique au quart environ (obtenue en mélangeant $20 \mathrm{ml}$ d'acide sulfurique pur et $80 \mathrm{ml}$ d'eau) et $5 \mathrm{ml}$ d'eau.

Placer le tube au bain-marie bouillant. Quand l'oxalate est entièrement dissous, titrer avec $\mathrm{KMnO4} 0,02 \mathrm{~N}$. La température doit rester supérieure à $60^{\circ} \mathrm{C}$ pendant le titrage.

$1 \mathrm{ml}$ de $\mathrm{KMnO} 040,02 \mathrm{~N}=0,4 \mathrm{mg}$ de Ca.

Soit $\mathrm{V}$ le volume de Permanganate employé.

La teneur du lait en calcium est exprimée par :

$0,0004 \times \mathrm{V} \times \frac{1000}{2}=0,2 \mathrm{~V}$ grammes de Ca par litre de lait,

ou $0,0004 \times \mathrm{V} \times \frac{100}{2}=0,02 \mathrm{~V}$ grammes de $\mathrm{Ca}$ pour $100 \mathrm{~g}$ de lait,

si les prises d'essai ont été respectivement et exactement $2 \mathrm{ml}$ ou $2 \mathrm{~g}$ de lait.

\section{2) Complexométrie.}

Après avoir centrifugé le précipité d'oxalate et éliminé le liquide surnageant (sans laver, ici, le culot de centrifugation) ajouter $5 \mathrm{ml}$ d'acide chlorhydrique $0,2 \mathrm{~N}$ chaud pour dissoudre le précipité d'oxalate puis ajouter $10 \mathrm{ml}$ de complexonate de magnésium disodique $0,01 \mathrm{~N}$ (c'est-à-dire à $0,43 \mathrm{p} .100$ dans l'eau) et $5 \mathrm{ml}$ d'eau. Refroidir.

Ajouter $1 \mathrm{ml}$ de $\mathrm{NaOH} \mathrm{N}$ et $2 \mathrm{ml}$ de tampon à $p \mathrm{H} 10(70 \mathrm{~g}$ de $\mathrm{NH}_{4} \mathrm{Cl}+570 \mathrm{ml}$ de $\mathrm{NH}_{4} \mathrm{OH}$ à $25 \mathrm{p} .100$ et diluer à $1000 \mathrm{ml}$ ).

Le $p \mathrm{H}$ du mélange doit être égal à 10 .

Ajouter 6 gouttes de solution de noir ériochrome $\mathrm{T}(0,5 \mathrm{~g}$ de noir ériochrome $+4,5 \mathrm{~g}$ de chlorhydrate d'hydroxylamine + alcool à 96 p. 100 pour dissoudre et compléter à $100 \mathrm{ml}$ ).

Titrer immédiatement avec une solution de complexon III (titriplex III) $0,01 \mathrm{~N}$ jusqu'à coloration bleu franc.

$1 \mathrm{ml}$ de complexon III (titriplex III) $0,01 \mathrm{~N}$ correspond à $0,4 \mathrm{mg}$ de Ca. 
Soit V le volume de complexon (titriplex) utilisé.

La teneur du lait en calcium est exprimée par :

$0,0004 \times \mathrm{V} \times \frac{1000}{2}=0,2 \mathrm{~V}$ grammes de Ca par 1 de lait,

ou $0,0004 \times \mathrm{V} \times \frac{100}{2}=0,02 \mathrm{~V}$ grammes de Ca pour $100 \mathrm{~g}$ de lait, si les prises d'essai ont été respectivement et exactement $2 \mathrm{ml}$ ou $2 \mathrm{~g}$ de lait.

\section{IV) Remarque.}

Dans les cas où l'on a utilisé pour l'analyse le filtrat de la défécation trichloracétique, les résultats obtenus sont trop élevés à cause du volume du précipité. Il faut les corriger en les multipliant par :

0,972 dans le cas du lait entier,

0,989 dans le cas du lait écrémé.

\section{$*^{*} *$ \\ DEUXIÈME PARTIE}

\section{Résultats obtenus}

(Voir tableau page suivante)

$$
*^{*} *
$$

\section{TROISIEMME PARTIE}

\section{Commentaires}

1) L'examen d'ensemble des 240 dosages effectués par 6 méthodes distinctes sur 20 laits différents montre que les résultats obtenus par ces diverses méthodes sont, dans la majorité des cas, très voisins les uns des autres pour un même lait. Dans quelques cas, il y a identité pratique entre les six résultats (12 analyses).

2) Il semble donc possible de conclure qu'il est indifférent d'employer pour la préparation de l'analyse : la défécation trichloracétique, la minéralisation par voie sèche ou par voie humide et, pour le dosage proprement dit : les méthodes manganimétrique ou complexométrique.

Cependant, en toute rigueur, les résultats moyens obtenus par la défécation trichloracétique, qui coïncident exactement avec la moyenne générale de tous les résultats, semblent devoir donner la préférence à cette méthode. 
Les résultats sont exprimés en $\mathrm{g}$ de calcium par 1 de lait.

Chaque valeur est la moyenne de deux déterminations.

\begin{tabular}{|c|c|c|c|c|c|c|c|}
\hline \multirow{2}{*}{$\begin{array}{c}\text { Nos des } \\
\text { échan- } \\
\text { tillons } \\
\text { (laits } \\
\text { de petit } \\
\text { mélange) }\end{array}$} & \multicolumn{2}{|c|}{$\begin{array}{c}\text { Défécation } \\
\text { trichloracétique }\end{array}$} & \multicolumn{2}{|c|}{$\begin{array}{l}\text { Minéralisation } \\
\text { voie sèche } \\
\text { (avec acétate } \\
\text { de magnésium) }\end{array}$} & \multicolumn{2}{|c|}{$\begin{array}{l}\text { Minéralisation } \\
\text { voie humide }\end{array}$} & \multirow{2}{*}{$\begin{array}{l}\text { Moyennes } \\
\text { des } \\
12 \text { analyses }\end{array}$} \\
\hline & $\begin{array}{l}\text { Mangani- } \\
\text { métrie }\end{array}$ & $\begin{array}{l}\text { Complexo- } \\
\text { métrie }\end{array}$ & $\begin{array}{l}\text { Mangani- } \\
\text { métrie }\end{array}$ & $\begin{array}{l}\text { Complexo- } \\
\text { métrie }\end{array}$ & $\begin{array}{l}\text { Mangani- } \\
\text { métrie }\end{array}$ & $\begin{array}{l}\text { Complexo- } \\
\text { métrie }\end{array}$ & \\
\hline 1 & 1,236 & 1,226 & 1,220 & 1,200 & 1,260 & 1,200 & 1,2237 \\
\hline 2 & 1,187 & 1,177 & 1,200 & 1,200 & 1,180 & 1,180 & 1,1873 \\
\hline 3 & 1,207 & 1,207 & 1,200 & 1,200 & 1,220 & 1,220 & 1,2090 \\
\hline 4 & 1,325 & 1,325 & 1,320 & 1,320 & 1,320 & 1,320 & 1,3217 \\
\hline 5 & 1,246 & 1,246 & 1,230 & 1,235 & 1,220 & 1,220 & 1,2330 \\
\hline 6 & 1,261 & 1,266 & 1,280 & 1,295 & 1,260 & 1,260 & 1,2703 \\
\hline 7 & 1,246 & 1,246 & 1,270 & 1,295 & 1,280 & 1,285 & 1,2703 \\
\hline 8 & 1,167 & 1,167 & 1,195 & 1,180 & 1,180 & 1,180 & 1,1781 \\
\hline 9 & 1,325 & 1,330 & 1,335 & 1,360 & 1,340 & 1,340 & 1,3366 \\
\hline 10 & 1,207 & 1,210 & 1,220 & 1,230 & 1,190 & 1,190 & 1,2078 \\
\hline 11 & 1,345 & 1,350 & 1,340 & 1,340 & 1,340 & 1,340 & 1,3425 \\
\hline 12 & 1,304 & 1,304 & 1,300 & 1,300 & 1,300 & 1,300 & 1,3013 \\
\hline 13 & 1,325 & 1,330 & 1,320 & 1,320 & 1,320 & 1,320 & 1,3225 \\
\hline 14 & 1,207 & 1,210 & 1,220 & 1,220 & 1,200 & 1,200 & 1,2095 \\
\hline 15 & 1,266 & 1,270 & 1,280 & 1,280 & 1,250 & 1,260 & 1,2676 \\
\hline 16 & 1,385 & 1,370 & 1,380 & 1,380 & 1,380 & 1,380 & 1,3791 \\
\hline 17 & 1,266 & 1,270 & 1,260 & 1,260 & 1,260 & 1,260 & 1,2626 \\
\hline 18 & 1,304 & 1,310 & 1,300 & 1,300 & 1,300 & 1,290 & 1,3006 \\
\hline 19 & 1,206 & 1,210 & 1,200 & 1,200 & 1,180 & 1,180 & 1,1960 \\
\hline 20 & 1,234 & 1,241 & 1,225 & 1,230 & 1,170 & 1,175 & 1,2125 \\
\hline Moyennes & 1,262 & 1,263 & 1,266 & 1,269 & 1,257 & 1,255 & 1,262 \\
\hline
\end{tabular}

3) La défécation trichloracétique est une méthode simple et rapide qui extrait la totalité du calcium du lait.

Il est très intéressant de constater que, après séparation du calcium à l'état d'oxalate, le dosage proprement dit de l'ion oxalate par manganimétrie ou celui de l'ion calcium par complexométrie conduisent à des résultats identiques.

Pour choisir entre ces deux méthodes de titrage, il convient de se baser sur les considérations suivantes :

a) Le titrage manganimétrique n'exige que deux réactifs simples et courants : acide sulfurique au quart et solution de permanganate titrée (d'usage courant dans tous les laboratoires).

b) Le titrage complexométrique exige six réactifs dont certains ne sont pas d'usage courant et doivent être spécialement préparés 
pour le dosage du calcium : acide chlorhydrique titré, complexonate de magnésium disodique, soude titrée, tampon à $p H 10$, solution de noir ériochrome $T$, solution de complexon III (ou titriplex III).

c) Le terme du dosage est plus facile à saisir dans le cas de la manganimétrie (apparition d'une coloration rose dans une liqueur incolore) que dans la complexométrie (passage du rouge violacé de plus en plus pâle au bleu pâle avec une légère incertitude au point exact de virage, même en lumière bleue). Le titrage complexométrique, pour être parfaitement utilisable, exige un certain entraînement de l'opérateur et risque de conduire à des résultats incorrects entre les mains d'une personne n'ayant pas l'habitude de cette méthode.

d) Le prix de revient du titrage complexométrique est plus élevé que celui du titrage manganimétrique (achat des réactifs, préparation des solutions).

e) Pour toutes ces raisons, nous donnons la préférence au titrage manganimétrique.

Remarque : l'utilisation de la défécation trichloracétique nécessite, comme nous l'avons dit plus haut, l'emploi d'un facteur de correction à cause du volume des précipités. Cela explique l'aspect particulier des chiffres obtenus par cette méthode dans le tableau précédent (alors que tous les autres résultats sont des multiples de 0,005).

4) La minéralisation par voie sèche (formation des cendres par calcination) conduit à d'excellents résultats.

Comme il nous l'a été demandé par le Groupe de travail, nous avons ajouté au lait de l'acétate de magnésium dans le but de prévenir une perte éventuelle de calcium. A vrai dire, et pour des raisons théoriques, cette perte n'était pas à craindre et l'adjonction d'un "fixateur" de calcium était théoriquement inutile. Nous avons néanmoins cherché à nous rendre compte de l'influence de ce sel et nous avons procédé à des dosages comparatifs de calcium sur des cendres préparées avec et sans addition d'acétate de magnésium.

Les résultats obtenus ont été identiques. Voici quelques exemples :

\begin{tabular}{|c|c|c|}
\hline & $\begin{array}{c}\text { Avec acétate } \\
\text { de } M g\end{array}$ & $\begin{array}{c}\text { Sans acétate } \\
\text { de } M g\end{array}$ \\
\hline $\begin{array}{l}\text { Echantillon A : } \\
\text { Titrage manganimétrique } \ldots \ldots \ldots \ldots \ldots \\
\text { Titrage complexométrique } \ldots \ldots \ldots \ldots\end{array}$ & $\begin{array}{l}1,240 \\
1,240\end{array}$ & $\begin{array}{l}1,240 \\
1,250\end{array}$ \\
\hline $\begin{array}{l}\text { Echantillon B : } \\
\text { Titrage manganimétrique } \ldots \ldots \ldots \ldots \ldots \\
\text { Titrage complexométrique } \ldots \ldots \ldots \ldots\end{array}$ & $\begin{array}{l}1,340 \\
1,320\end{array}$ & $\begin{array}{l}1,340 \\
1,320\end{array}$ \\
\hline
\end{tabular}


Dans la grande majorité des cas, les résultats obtenus par les deux méthodes de titrage (manganimétrie et complexométrie) ont été identiques.

Pour les raisons indiquées plus haut, nous donnons cependant la préférence à la méthode de dosage par manganimétrie.

Une question se pose maintenant : faut-il, ou non, préférer la minéralisation par voie sèche à la défécation trichloracétique ?

Il est bien évident que si, sur un échantillon de lait, on doit déterminer à la fois les cendres et le calcium, on dosera le calcium sur les cendres (obtenues sans addition d'acétate de magnésium) sans recourir à la défécation T.C.A.

En revanche, la méthode par défécation T.C.A permet d'obtenir des résultats beaucoup plus rapidement et se prête mieux à des dosages en série.

N'ayant, ici, en vue que le dosage du calcium, nous choisirons et recommanderons la défécation trichloracétique (suivie du titrage manganimétrique).

5) La minéralisation par voie humide (nitroperchlorique) ne nous était pas demandée par le Groupe de travail pour le dosage $\mathrm{du}$ calcium.

Nous l'avons néanmoins expérimentée à titre de curiosité car nous avons eu à étudier la minéralisation nitroperchlorique pour le dosage du phosphore (qui fait l'objet d'un rapport indépendant) et nous voulions savoir si, dans le cas où l'on aurait à doser à la fois le calcium et le phosphore sur un même échantillon de lait, un mode d'attaque unique ne pourrait pas éventuellement convenir aux deux dosages.

L'expèrience a montré que les résultats obtenus par cette méthode de minéralisation étaient parfaitement comparables à ceux des deux autres méthodes.

D'autre part la manganimétrie et la complexométrie conduisent, là encore, à des chiffres pratiquement identiques entre eux.

Cette méthode de minéralisation n'est cependant pas sans inconvénients : elle exige plus de surveillance que les autres et, dans certains cas, elle peut entraîner des accidents.

Pour toutes ces raisons, (jointes au fait que son étude n'était pas demandée par le Groupe de travail), nous n'en recommanderons pas l'emploi.

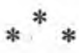




\section{QUATRIEMME PARTIE}

\section{Conclusion : choix d'une méthode}

Pour les raisons indiquées dans les commentaires précédents, nous proposons, pour le dosage du calcium dans le lait, une méthode analytique basée sur les principes suivants :

1) Défécation trichloracétique pour mettre le calcium du lait en solution et insolubiliser les protides que l'on sépare par filtration.

2) Séparation du calcium à l'état d'oxalate : le calcium est précipité dans le sérum déprotéiné à l'état d'oxalate et séparé par centrifugation.

3) Titrage de l'oxalate de calcium par manganimétrie : le précipité d'oxalate séparé et lavé est dissous et l'ion oxalate est dosé par une solution titrée de permanganate.

Le détail de cette technique a été finalement adopté par la Commission des analyses chimiques de la F.I.L. et publié comme « Norme Officielle Internationale de la Fédération Internationale de Laiterie " (année 1966. Norme $n^{\circ} 36$ ) dont nous reproduisons ci-après le texte intégral.

$$
* * *
$$

NORME INTERNATIONALE F.I.L. no 36 (1966)

\section{Détermination de la teneur en calcium du lait}

\section{1) Objet.}

Cette méthode est applicable à tous les laits liquides normaux ainsi qu'aux laits reconstitués par dilution ou dissolution des laits concentrés ou des laits secs.

\section{2) Définition de la teneur en calcium du lait.}

Par teneur en calcium du lait, il faut entendre la quantité totale de calcium, exprimée en pourcentage pondéral, que l'on obtient lors de l'application de la méthode décrite ci-après.

\section{3) Principe de la méthode.}

La totalité du calcium du lait est mise en solution et les matières protéiques sont précipitées par l'acide trichloracétique. Le calcium contenu dans le filtrat est précipité sous forme d'oxalate de calcium, qui est séparé par centrifugation et titré à l'aide de permanganate de potassium.

\section{4) Réactifs.}

4.1 Acide trichloracétique : solution aqueuse à 20 p. 100 (poids/volume).

4.2 Acide trichloracétique : solution aqueuse à 12 p. 100 (poids/volume).

4.3 Oxalate d'ammonium : solution aqueuse saturée è froid. 
4.4 Rouge de méthyle : solution à 0,05 p. 100 (poids/volume) dans l'alcool éthylique à $96 \mathrm{p} .100$ (volume/volume).

4.5 Acide acétique : solution aqueuse à 20 p. 100 (volume/volume).

4.6 Ammoniaque : solution aqueuse obtenue en mélangeant à volumes égaux de l'ammoniaque à 25 p. 100 (poids/poids) et de l'eau distillée.

4.7 Ammoniaque : solution aqueuse obtenue en diluant $2 \mathrm{ml}$ d'ammoniaque à 25 p. 100 (poids/poids) à $100 \mathrm{ml}$ avec de l'eau distillée.

4.8 Acide sulfurique : solution aqueuse obtenue en ajoutant $20 \mathrm{ml}$ d'acide sulfurique à $98 \mathrm{p} .100$ (poids/poids) à $80 \mathrm{ml}$ d'eau distillée.

4.9 Permanganate de potassium : solution aqueuse titrée, $0,02 \mathrm{~N}$.

Tous les réactifs doivent être de qualité analytique.

\section{5) Appareillage.}

5.1 Balance analytique.

5.2 Fiole jaugée de $50 \mathrm{ml}$.

5.3 Pipette à lait de $20 \mathrm{ml}$.

5.4 Papier filtre sans cendre pour filtration lente.

5.5 Centrifugeuse pouvant développer une accélération centrifuge égale à $1400 \mathrm{~g}$.

5.6 Tubes de centrifugeuse cylindriques à fond rond, de $30 \mathrm{ml}$ environ, marqués à $20 \mathrm{ml}$.

5.7 Pipettes de 2 à $5 \mathrm{ml}$.

5.8 Dispositif de siphonnage à succion muni d'un tube capillaire.

5.9 Bain-marie bouillant.

5.10 Burette graduée en $1 / 50 \mathrm{ml}$.

\section{6) Echantillonnage.}

Se reporter à la norme internationale FIL/IDF $2: 1958$ "Méthodes pour le prélèvement d'échantillons de lait et de produits laitiers \$ (dernière édition).

\section{7) Mode opératoire.}

\subsection{Préparation de l'échantillon.}

Avant l'analyse, porter l'échantillon à $20 \pm 2^{\circ} \mathrm{C}$ et mélanger soigneusement. Si l'on n'obtient pas une dispersion homogène de la matière grasse, chauffer l'échantillon lentement à $40^{\circ} \mathrm{C}$, mélanger doucement et refroidir à $20^{\circ} \pm 2^{\circ} \mathrm{C}$.

7.2 Défécation de la prise d'essai.

7.2.1 Dans une fiole jaugée de $50 \mathrm{ml}$, peser à $10 \mathrm{mg}$ près environ ᄂ $20 \mathrm{~g}$ de lait.

7.2.2 Ajouter peu à peu en agitant une solution aqueuse d'acide trichloracétique à 20 p. 100 et compléter à $50 \mathrm{ml}$ avec ce réactif. Agiter vigoureusement pendant quelques secondes. Laisser reposer 30 minutes.

7.2.3 Filtrer sur papier filtre sans cendres. Le filtrat obtenu doit être limpide.

7.3 Précipitation du calcium à l'état d'oxalate et séparation de l'oxalate.

7.3.1 Dans un tube de centrifugeuse cylindrique à fond rond introduire $5 \mathrm{ml}$ du filtrat limpide $(7.2 .3)$, puis $5 \mathrm{ml}$ d'acide trichloracétique à 12 p. $100,2 \mathrm{ml}$ d'une solution aqueuse saturée d'oxalate d'ammonium, 2 gouttes de solution alcoolique de rouge de méthyle et $2 \mathrm{ml}$ d'acide acétique à $20 \mathrm{p} .100$.

7.3.2 Mélanger par agitation circulaire et ajouter peu à peu de la solution d'ammoniaque (4.6) jusqu'à coloration jaune pâle, puis quelques gouttes d'acide acétique à 20 p. 100 jusqu'à coloration rose. 
7.3.3 Laisser reposer 4 heures à la température ordinaire.

7.3.4 Diluer à $20 \mathrm{ml}$ avec de l'eau et centrifuger $10 \mathrm{mn}$ à $1400 \mathrm{~g}$.

7.3.5 Décanter le liquide clair surnageant avec un dispositif à succion.

7.3.6 Rincer les parois du tube de centrifugation (sans remettre en suspension le culot d'oxalate de calcium) avec $5 \mathrm{ml}$ de solution d'ammoniaque diluée (4.7). Centrifuger $5 \mathrm{mn}$ à $1400 \mathrm{~g}$.

7.3.7 Décanter le liquide surnageant avec le dispositif à succion.

7.3.8 Procéder à trois lavages successifs.

7.4 Titrage de l'oxalate.

7.4.1 Après avoir enlevé par siphonnage la dernière eau de lavage, ajouter sur le culot d'oxalate de calcium $2 \mathrm{ml}$ d'une solution aqueuse d'acide sulfurique (4.8) et $5 \mathrm{ml}$ d'eau distillée.

7.4.2 Placer le tube au bain-marie bouillant et, quand l'oxalate est entièrement dissous, le titrer avec une solution de permanganate de potassium $0,02 \mathrm{~N}$, jusqu'à coloration rose persistante. La température doit rester supérieure à $60^{\circ} \mathrm{C}$ pendant le titrage.

\subsection{Essai à blanc.}

Effectuer un essai à blanc avec tous les réactifs en remplaçant la prise d'essai de lait par $20 \mathrm{ml}$ d'eau distillée.

\section{8) Expression des résultats.}

\subsection{Mode de calcul.}

Calculer la teneur en calcium à l'aide de la formule suivante : Teneur en calcium p. $100=$

$$
0.0004 \times(\mathrm{V}-\mathrm{v}) \times \frac{1000}{\mathrm{P}} \times \mathrm{K}=0.4 \times(\mathrm{V}-\mathrm{v}) \times \frac{\mathrm{K}}{\mathrm{P}}
$$

dans laquelle :

$\mathrm{V}=$ nombre de $\mathrm{ml}$ de $\mathrm{KMnO}_{4}(0,02 \mathrm{~N})$ utilisés dans le titrage de la prise d'essai.

$\mathrm{v}=$ nombre de $\mathrm{ml}$ de $\mathrm{KMnO}_{4}(0,02 \mathrm{~N})$ utilisés dans l'essai à blanc.

$\mathrm{P}=$ poids en grammes de la prise d'essai initiale (environ 20 g.).

$\mathrm{K}=$ coefficient de correction pour le volume du précipité résultant de la défécation trichloracétique :

Pour le lait entier :

$(3,5$ à 4,5 p. 100 de matière grasse $): K=0,972$.

Pour du lait à :

3 p. 100 de matière grasse : $K=0,976$.

Pour du lait à :

2 p. 100 de matière grasse : $K=0,980$.

Pour du lait à :

1 p. 100 de matière grasse : $K=0,985$.

Pour du lait écrémé : $\mathrm{K}=0,989$.

\subsection{Répétabilité des résultats.}

La différence entre les résultats de deux déterminations effectuées simultanément ou rapidement l'une après l'autre par le même analyste, ne doit paș excéder $0,002 \mathrm{~g}$ de calcium pour $100 \mathrm{~g}$ de lait. 


\section{Summary}

The International Dairy Federation has standardized an official method for the determination of calcium in milk on the following basis :

1) Defecation of a test portion of $20 \mathrm{~g}$ with trichloracetic acid.

This treatment of the test portion was preferred to dry ashing and to wet mineralization. The reasons for that are given.

2) Precipitation of calcium, on an aliquot part of the filtrate, as oxalate; separation of the oxalate by centrifugation and washing of the precipitate.

3) After dissolution of this calcium oxalate in diluted sulfuric acid, titration of the oxalate with a solution of potassium permanganate. This treatment was preferred to complexometric titration for the given reasons.

The repeatibility of this method is excellent (less than $0,002 \mathrm{~g}$ of calcium for $100 \mathrm{~g}$ of milk). 